\title{
DIALOGO ABERTO: UM MÉTODO PARA ENFRENTAMENTO DA PSICOSE
}

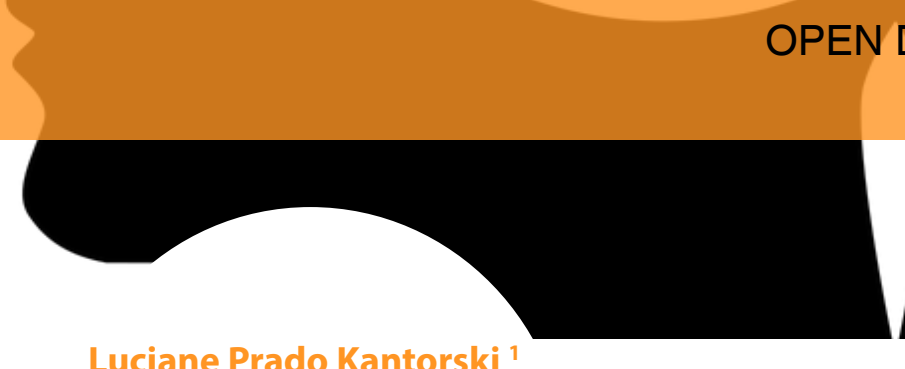

Luciane Prado Kantorski

Mario Mario Cardano ${ }^{2}$

RESUMO

O Método do Diálogo Aberto surgiu no oeste da Lapônia, no início dos aos 80 como uma forma de atenção imediata à crise psicótica. Este artigo tem como objetivo apresentar uma análise do Método do Diálogo Aberto para o enfrentamento de crises psicóticas em seus fundamentos teóricos, aplicação prática e resultados. Neste artigo é apresentada uma análise do Método do Diálogo Aberto sendo discutido: O diálogo aberto - histórico e princípios (ajuda imediata, perspectiva de rede social, flexibilidade e mobilidade, responsabilidade, continuidade psicológica, tolerância a incerteza, dialogismo).

Palavras chave: Transtornos psicóticos. Saúde mental. Rede social. Relações familiares.

\section{ABSTRACT}

The Open Dialogue Method was developed in West Lapland in the beginning of the 1980s as an immediate care approach to psychotic crises. This article aims to present an analysis of the Open Dialogue Method in order to face psychotic crises as to its theoretical foundations, practical application and

\footnotetext{
${ }^{1}$ Professora Associada da Faculdade de Enfermagem da Universidade Federal de Pelotas. E-mail: kantorski@ pq.cnpq.br

${ }^{2}$ Instituição/Afiliação Università Degli Studi di Torino, Itália. E-mail: mario.cardano@ unito.it
} 
results. It is a theoretical essay about an analysis of the Open Dialogue in which are discussed: The Open Dialogue - history and its principles (immediate help, social network perspective, flexibility and mobility, responsibility, psychological continuity, tolerance of uncertainty, dialogism).

Keywords: Psychotic disorders. Mental health. Social network. Family relationships.

\section{INTRODUÇÃOO}

Nos últimos dezesseis anos temos liderado estudos e pesquisas do Grupo de Pesquisa cadastrado no CNPq, Enfermagem em Saúde Mental e Coletiva no campo da avaliação em saúde mental, com grandes projetos de pesquisa aprovados e executados, financiados pelo CNPq e Ministério da Saúde como a Avaliação dos Centros de Atenção Psicossociais do sul do Brasil I (CAPSUL I) - desenvolvido entre 2005 e 2008 e CAPSUL II - 2011 a 2016 e Redes que reabilitam - avaliando experiências inovadoras de composição de inovadoras de composição de redes de atenção psicossocial (REDESUL) - desenvolvido entre 2008 e 2010. A avaliação de serviços em saúde mental tem sido o foco da contribuição de nossas pesquisas para o campo das políticas de saúde no Brasil, no contexto da reforma psiquiátrica e da desinstitucionalização.

No entanto, a experiência da reforma psiquiátrica brasileira apresenta importantes limites decorrentes da concomitante presença dos hospitais psiquiátricos na rede de saúde. Mesmo com a inversão do financiamento (deslocamento de parte do financiamento público dos leitos em hospitais psiquiátricos para os serviços da rede de atenção psicossocial), as práticas institucionalizantes em relação à loucura persistem de diversas formas e se objetivam em entraves para o processo de reforma.

A política de saúde mental brasileira tem muita influência da psiquiatria democrática Basagliana, a partir das experiências italianas e da Lei de Reforma Psiquiátrica Italiana (Lei 180) aprovada em 1978.

A possibilidade de realização do pós-doutorado na Itália, no ano de 2015, constituiu-se numa oportunidade de aprofundamento, de preencher lacunas, de consolidar parcerias, de constituir redes de pesquisa no campo da saúde mental. Permitiu ainda um importante avanço na formação como pesquisadora no campo das políticas de saúde mental em contextos desinstitucionalizantes como a realidade italiana, berço da reforma psiquiátrica no mundo.

Durante o ano de 2015 e inicio de 2016 os estudos realizados junto ao Dipartimento di Culture, Politica e Società da Università Degli Studi di Torino na Itália sob supervisão do Prof. Dr. Mario Cardano, este com expertise em pesquisa qualitativa no campo da saúde mental e das políticas 
de saúde, possibilitaram a aproximação com o Diálogo Aberto e seu mentor, Jaako Seikkula. O Prof. Dr. Mario Cardano vem desenvolvendo suas pesquisas com o foco em duas áreas: primeiro, as ciências sociais com ênfase em métodos e epistemologia da pesquisa qualitativa e se-gundo, sociologia da saúde, especificamente saúde mental ("desability"), abordando temas como desigualdade social; trabalho, habitação, cidadania e sua relação com a saúde mental. Particularmente estes estudos que articulam as políticas de saúde (sanitárias) às políticas não sanitárias (como de trabalho, de habitação) na região de Piemonte - Itália se inserem neste campo de interesse das políticas de saúde/saúde mental e suas interfaces.

A pesquisa realizada a partir de 2007 pelo Prof. Dr. Mario Cardano e por Giulia Lepori, que se propõe a reconstruir a experiência do Grupo Voci di Settimo Torinese (que se constituiu em 2001), não como grupo terapêutico, mas como grupo de pesquisa sobre as vozes, sistematizada no livro "Udirelavoce degli dei. L'esperienza del gruppo você (CARDANO; LEPORI, 2012) contribui para ampliar a perspectiva de pesquisas no campo das práticas de saúde mental no território que escapam dos processos de medicalização da vida.

O movimento dos ouvidores de vozes "Intervoice", do qual MariusRomme e Sandra Escher são fundadores; representa um empenho em desmedicalizar a experiência de ouvir vozes e se difunde a partir do início dos anos noventa, a partir do qual se constituiu uma rede interna-cional em países como a Holanda, Áustria, Inglaterra, Escócia, País de Gales, Irlanda, França, Suécia, Noruega, Espanha, Itália, Suíça, Alemanha, Dinamarca, Grécia, Austrália, Nova Zelândia. Na Itália a difusão do grupo de ouvidores de vozes ocorreu em Lodi, Milano, Prato, Reggio-Emilia, Roma, Settimo Torinese, Venaria, Trento e Trieste (CARDANO; LEPORI, 2012).

Em janeiro de 2015 constituiu-se a primeira oportunidade de contato com o Diálogo Aberto viabilizado pelo Prof, Dr, Mario Cardano que integra o Projeto intitulado: Diálogo Aberto - uma abordagem inovadora no tratamento das crises psiquiátricas - formulação e avaliação de ferramentas organizacionais para a transferibilidade do "Diálogo Aberto" no Departamento de Saúde Mental italiano. Este projeto é coordenado cientificamente pela Dra. Maria Chiara Rossi e envolve 8 Departamentos de Saúde Mental na Itália nas cidades de Turim (dois departamentos ex-istentes), Savona, Trieste, Modena, Roma (dois departamentos de cinco) e Catania. O projeto irá adaptar o método para a estrutura organizacional dos departamentos de saúde mental, selecionar e treinar trabalhadores de saúde para formar as equipes móveis, para tratar com o Diálogo Aberto os novos casos de crises psiquiátricas e avaliar a eficácia terapêutica em todos os indivíduos tratados no período (até 2017).

Em 20 de fevereiro de 2015, em Turim, teve-se a oportunidade de participar de um momento de formação com Jaakko Seikkula, PHD, Pro- 
fessor de Psicoterapia do Departamento de Psicologia da University of Jyväskylä na Finlândia, o criador do Diálogo Aberto.Este artigo tem como objetivo apresentar o Método do Diálogo Aberto utilizado na Finlândia para o enfrentamento de crises psicóticas em seus principais fundamentos.

\section{Histórico do Diálogo Aberto}

O Método do Diálogo Aberto surgiu na Lapônia, no início dos anos 80 como uma forma de atenção imediata a crise psicótica e a outras crises graves, ou seja, uma prática psiquiátrica em que o cuidado a crise é organizado durante as 24 horas sucessivas ao primeiro contato. Na qual o paciente e sua família são convidados a participar não só do primeiro encontro, mas de todo processo de tratamento. O idealizador deste método Jaakko Seikkula, psicólogo clínico começou a trabalhar no hospital de Keropudas - Tornio em 1981, e na sequência contou com os médicos Jyrki Keranen e Birgitta Alakare, os enfermeiros, Ilkka Vehkaperä e Telma Hihnala, e os psicólogos Kauko Haarakangas, Markku Sutela,esta equipe iniciou a aplicação do método. Quando iniciou-se a trabalhar com este sistema de tratamento, o foco de interesse eram pacientes com diagnóstico de esquizofrenia porque o hospital de Keropudas atendia muitos pacientes com esquizofrenia. $O$ professor Alanen e sua equipe desde 0 final dos anos 60 implantaram o Turku Schizophrenia Project que era um projeto de pesquisa e intervenção voltado para o paciente esquizofrênico e sua família e visava desenvolver um modelo de tratamento compreensivo e psicoterapeuticamente orientado, aplicado a assistência psiquiátrica pública. Entre 1981 e 1987 implantou-se o Finnish National Schizophrenia Project, que consistia de um programa nacional para o estudo, tratamento e reabilitação de pacientes esquizofrêncios que se utilizava do modelo psicoterapeutico desenvolvido em Turku e que visava diminuir a hospitalização e privilegiar a assistência ambulatorial, demosntrando sua aplicabilidade no sistema público de cuidado (SEIKKULA, 2011).

Na Finlândia, a prática psicoterápica tem sido uma parte da saúde pública importante desde o final dos anos 60, com o trabalho do professor Yrjö Alanen e sua equipe iniciandocom a psicoterapia psicodinâmica individual. A equipe de Turku no final de 1970 enfatizava que cada processo de tratamento é único e deveria ser adaptado às diferentes necessidades de cada paciente. Os aspectos inovadores desta abordagem concentram-se em: intervenção precoce e rápida em todos os casos; o planejamento do tratamento para atender a mudança; identificação de necessidades específicas de cada paciente e da família, integrando métodos terapêuticos em um único processo de tratamento; tendo uma atitude terapêutica como orientação básica para cada membro da equipe, ainda percebendo o tratamento como um processo contínuo e monitorando o seu progresso e os 
seus resultados (SEIKKULA, 2011).

O modelo "Need-Adapted Treatmentof Psychosis" desenvolvido por Alanen tem como princípios uma especialização horizontal e a desritualização do tratamento. Neste modelo os psiquiatras trabalham como parte de uma equipe multiprofissional e os aspectos do tratamento são discutidos e decididos em conjunto com pacientes, familiares e equipe durante as reuniões de terapia, enfocando-se as experiências dos pacientes e evitando o domínio de uma perspectiva médica que pode envolver tratamentos desnecessários. Um dos objetivos é adiar a administração de neurolépticosna fase inicial. A base da tradição des-ta abordagem não é o controle imediato dos sintomas psicóticos, mas a formação de um diálogocom os pacientes e outros participantes nas reuniões de terapia. A abordagem do Diálogo Aberto consiste numa uma modificação do "Need-Adapted Treatment of Psychosis" e foi sistematicamente desenvolvida desde o início da década de 1980 na Finlândia (BORCHERS, 2014).

Numa revisão de literatura os estudos sobre os resultados e documentos descritivos publicados em idioma Inglês associados ao Diálogo Aberto indicam que, em pequenos grupos de pessoas no oeste da Lapônia a duração da psicose sem tratamento foi reduzida; que a maioria das pessoas conseguiu uma recuperação funcional com o mínimo de uso de medicamentos antipsicótios e têm poucos sintomas residuais (LAKEMAN ,2014).

\section{O Diálogo Aberto e seus princípios}

Os fundamentos do Método Diálogo Aberto consistem essencialmente em realizar a primeira reunião de tratamento dentro de 24 horas após o primeiro contato e incluir tantas pessoas significativas quanto possível da rede social do paciente. O objetivo central consiste em gerar diálogo e colocar em palavras as experiências vivenciadas pelo paciente na crise psicótica. Todas as questões são analisadas e tratadas com todos presentes, ou seja, nenhuma questão é tratada na ausência do paciente e sua família. $O$ tratamento é adaptado as necessidades específicas do paciente e ocorre em casa, sempre que possível. A continuidade psicológica é garantida através da construção de equipes integradas voltadas para geração de diálogo com a família e os pacientes, em vez de tentar remover rapidamente os sintomas psicóticos (SEIKKULA, 2014; ARNKIL; SEIKKULA, 2012; SEIKKULA, 2003). Já em 1984 o encontro aberto com o paciente, a família, sua rede social, outros profissionais era nesta região uma prática padrão e haviam integrado a esta prática uma visão sistêmica da família e do paciente psicótico. O método não foi criado teoricamente, mas desenvolvido gradualmente na prática, a partir dos problemas que emergiam e da busca de possíveis soluções para os mesmos. Os próprios en- 
contros de tratamento constituiram-se em oportunidades de construções conjuntas, de trabalho em equipe, de busca de soluções, e percebeu-se que havia uma preciosa potencialidade para recuperação do paciente no interior de sua rede social (SEIKKULA, 2014).

A filosofia central do Método do Diálogo Aberto é o desenvolvimen-to de relações entre as pessoas que estão enfrentando problemas, a família e a rede social envolvida, e ofertar apoio a pessoa em casa, ao invés de instituições ou locais de reabilitação. São mais valorizadas as habilidades dos ennvolvidos de gerar diálogos em reuniões conjuntas, mantendo a comunicação aberta entre as partes, respeitando as vozes de todos os participantes, a paciência de ouvir e de não tomar decisões precipitadas, a capacidade de constantemente enfatizar a situação familiar e as questões da vida cotidiana (BORG et al., 2009).

Seikkula, Alakare e Aaltonen (SEIKKULA et al., 2001) após vários programas de investigação e formação em psicoterapia, elencaram sete princípios principais do tratamento com o Método do Diálogo Aberto que são:

1. A ajuda imediata. As unidades de atendimento devem organizar a primeira reunião dentro de 24 horas após o primeiro contato com o paciente, contando com um familiar e profissionais de referência da instituição. A resposta imediata tem como finalidade evitar a hospitalização em tantos casos quanto possível. O paciente psicótico participa desde as primeiras reuniões durante o período mais intenso da crise psicótica.

2. A perspectiva de rede social. Os pacientes, suas famílias, e outros membros significativos de sua rede social são sempre convidados para as reuniões para mobilizar apoio para o paciente e a família. Os outros membros-chave podem ser representantes institucionais, de serviços de reabilitação profissional, colegas de trabalho, vizinhos ou amigos.

3. Flexibilidade e mobilidade. Estes devem ser garantidos através da adaptação do tratamento às necessidades específicas de cada caso, utilizando os métodos terapêuticos adequados a cada situação. As reuniões de tratamento são com a aprovação da família, organizadas preferencialmente no casa do paciente.

4. Responsabilidade. O profissional que é contactado pela família é responsável por organizar a primeira reunião. A seguir as decisões são tomadas nas reuniões havendo uma equipe que se encarrega de todo o tratamento.

5. Continuidade psicológica. A equipe assume a responsabilidade pelo tratamento por tanto tempo quanto necessário. Os representantes da rede social do paciente participam nas reuniões de tratamento durante toda a seqüência de tratamento, incluindo, quando são aplicados outros métodos terapêuticos.

6. Tolerância a incerteza. Consiste em estabelecer um sentimento de confiança para fortalecer o processo de articulação. Em crises psicóti- 
cas, um sentido de segurança adequado pressupõe encontros frequentes, por vezes, todos os dias pelo menos para os primeiros 10-12 dias. Depois disso, as reuniões são organizadas em uma base regular de acordo com os desejos da família. Normalmente nenhum contrato terapêutico detalhado é feito no período de crise, evitando decisões precipitadas. Por exemplo, o uso de neurolépticos não é iniciado na primeira reunião, em vez disso, a sua pertinência deve ser discutida em, pelo menos, três reuniões antes da implementação.

7. Dialogismo. O foco está principalmente na promoção do diálogo e secundariamente na promoção da mudança no paciente ou na família. $O$ Diálogo é visto como um fórum através do qual as famílias e os pacientes são capazes de discutir e buscar solução para os problemas, construindo novos entendimentos.

Doze elementos-chave da prática dialógica no Diálogo Abertosão elencados enquanto critérios de fidelidade, que são: participação de dois (ou mais) terapeutas na reunião; envolvimento da família e rede social; usar perguntas abertas; responder às coisas ditas pelo paciente; enfatizar o momento; solicitar múltiplos pontos de vista; usar o foco relacional no diálogo; responder aos problemas dialógicos e comporta-mentais com um estilo concreto e atento aos significados; enfatizar as palavras usadas pelo paciente e sua história, não os sintomas; a conversa entre profissionais (reflexões) deve ocorrer nas reuniões de tratamento; ser transparente; tolerar a incerteza (OLSON et al., 2014).

No oeste da Lapônia algumas escolhas estratégicas foram feitas, a fim de assegurar a continuidade dos cuidados. Nos serviços de internação (estão dispníveis 30 leitos hospitalares de cuidados agudos) e 5 clínicas ambulatoriais em que o Diálogo Aberto é a filosofia de prática, e em cada unidade, equipes móveis para atendimento a crise estão disponíveis. A maioria dos profissionais também são treinados (por cerca de 3 anos) em terapia familiar sistêmica. No modelo de Diálogo Aberto o diagnóstico não é um dado especifico que define a organização da atenção. Se o tratamento hospitalar para um novo usuário do serviço for considerado necessário, a equipe assume a responsabilidade de todo o processo de tratamento, independentemente do indivíduo ficar em casa ou no hospital, e independentemente de quanto tempo necessitar de ajuda e apoio (BORG et al., 2009).

Robert Whitaker em seu livro que se tornou um bestseller "Anatomy of an epidemic: Magic bullets, psychiatric drugs, and the astonishing rise of mental illness in America" fala de todos os danos que foram produzidos na América do Norte pelo uso massivo de psicofármacos em resposta as situações de estresse. No final de sua obra, o autor apresenta alguns projetos, dentre eles o Diálogo Aberto da Finlândia, como possíveis soluções a este grave contexto. $O$ autor conheceu a experiência e acompanhou o grupo da Lapônia e em seu livro fazuma descrição das bases do método 
e apresenta alguns resultados das pesquisas do grupo de Seikkula que comprovam a eficácia do mesmo (WHITAKER, 2010).

Os estudos de resultados da aplicação do Método do Diálogo Aberto em pacientes em primeira crise psicótica e em tratamento com o Diálogo Aberto, estudos de follow up em dois, cinco e dez anos incluindo grupos de controle (AALTONEN et al., 2011; SEIKKULA, 2013; SEIKKULA, 2006; SEIKKULA et al., 2011), demonstram que os pacientes que utilizaram do Método do Diálogo Aberto ficaram hospitalizados por menor número de dias e que os psicofármacos foram utilizados em menor número de casos.

Os resultados obtidos com o Diálogo Aberto nas últimas décadas na Finlândia comprovam a necessidade de dedicar tempo, trabalho e estudos sobre o método, reconhecendo que este vem cumprindo um importante papel no enfrentamento da psicose, no manejo adequado de psicofármacos, na cronicidade, na inserção social das pessoas.

\section{REFERÊNCIAS}

AALTONEN, J.; SEIKKULA, J.;LEHTINENB, K. The comprehensive open-dialogue approach in western Lapland I -the incidence of non-affective psychosis and prodromal states. Psychosis, v. 3, n. 3, p. 179-191, 2011. DOI: $10.1080 / 17522439.2011 .601750$

ARNKIL, T. E.;SEIKKULA, J. Metodo dialogici nel lavoro di rete: per la psicoterapia di gruppo, il servizio sociale e la didattica. Trento: Erickson, 2012.

BORCHERS, P. The need-adapted treatment of psychosis and the psychiatrist's inner dialogue. Jyväskylä: University of Jyväskylä, 2014. p. 1387.

BORG, M. et al.Implementing person-centered care in psychiatric rehabilitation: what does this involve? Isr J Psychiatry Relat. Sci.,v. 46, n. 2, p. 84-93, 2009.

CARDANO, M.; LEPORI, G. Udire la vocedegli dei: l'esperienza del gruppovoci. Milano, Franco Angeli, 2012.

LAKEMAN, R. The finnish open dialogue approach to crisis intervention in psychosis: a review. Psychotherapy in Australia. v.20, n.3, p. 26-33, 2014.

OLSON, M.;SEIKKULA, J.;ZIEDONIS, D. The key elements of dialogic practice: in open dialogue: fidelity criteria. Version 1.1: Disponível em: 
$<$ http://umassmed.edu/psychiatry/globalinitiatives/opendialogue/> Acesso em: 2 set. 2014.

SEIKKULA, J.;ALAKARE, B.;AALTONEN, J. Open dialogue in psychosis I: an introduction and case illustration. Journal of Constructivist Psychology, v. 14, n. 4, p. 247-265, 2001.DOI: 10.1080/10720530125965

SEIKKULA, J. et al. Open dialogue approach: treatment principles and preliminary results of a two- year follow-up on first episode schizophrenia. Ethical and Human Sciences and Services, v. 5, n. 3, p. 163-182, 2003.

SEIKKULA, J. Open dialogue integrates individual and systemic approaches in serious psychiatric crises. Smith College Studies in Social Work, v. 73, n. 2, p.227-245, 2003.

SEIKKULA, J. et al. Five-year experience of first-episode non affective psychosis in open-dialogue approach: Treatment principles, follow-up outcomes, and two case studies. Psychotherapy Research, v. 16, n. 2, p. 214-228, 2006. DOI: 10.1080/10503300500268490

SEIKKULA, J.; ALAKAREB, B.;AALTONENA, J. The comprehensive open-dialogue approach in western Lapland II - long-term stability of acute psychosis outcomes in advanced community care. Psychosis, v. 3, n. 3, p. 192-204, 2011.DOI: 10.1080/17522439.2011.595819

SEIKKULA, J. Becoming dialogical: psychotherapy or a way of life? The Australian and New Zealand Journal of Family Therapy. v.32, n.3, p. 179-193, 2011.

II dialogo aperto: l'approccio finlandese alle gravi crisi psichiatriche. Roma: Giovani Fioriti, 2014.

WHITAKER. R. Anatomy of an epidemic: magic bullets, psychiatric drugs, and the astonishing rise of mental illness in America. New York: Crown, 2010.

Data de recebimento: 10 de dezembro de 2016.

Data de aceite para publicação: 03 de maio de 2017. 